\title{
Engineering a novel self-powering electrochemical biosensor
}

\author{
X. Gu $\cdot$ M. Trybiło $\cdot$ S. Ramsay $\cdot$ M. Jensen $\cdot$ \\ R. Fulton $\cdot$ S. Rosser $\cdot$ D. Gilbert
}

Received: 11 December 2009/Revised: 21 July 2010/Accepted: 1 September 2010/Published online: 18 September 2010

(C) The Author(s) 2010. This article is published with open access at Springerlink.com

\begin{abstract}
This paper records the efforts of a multi-disciplinary team of undergraduate students from Glasgow University to collectively design and carry out a 10 week project in Synthetic Biology as part of the international Genetic Engineered Machine competition (iGEM). The aim of the project was to design and build a self-powering electrochemical biosensor called 'ElectrEcoBlu'. The novelty of this engineered machine lies in coupling a biosensor with a microbial fuel cell to transduce a pollution input into an easily measurable electrical output signal. The device consists of two components; the sensor element which is modular, allowing for customisation to detect a range of input signals as required, and the universal reporter element which is responsible for generating an electrical signal as an output. The genetic components produce pyocyanin, a competitive electron mediator for microbial fuel cells, thus enabling the generation of an electrical current in the presence of target chemical pollutants. The pollutants tested in our implementation were toluene and salicylate. ElectrEcoBlu is expected to drive forward the development of a new generation of biosensors. Our approach exploited a range of state-of-the-art modelling techniques in a unified framework of qualitative, stochastic and continuous approaches to support the design and guide the construction of this novel biological machine. This work shows that integrating engineering techniques with scientific methodologies can provide new insights into genetic regulation
\end{abstract}

\footnotetext{
M. Trybiło $(\bowtie) \cdot$ D. Gilbert

School of Information Systems, Computing and Mathematics, Brunel University, Uxbridge, Middlesex UB8 3PH, UK e-mail:maciej.trybilo@brunel.ac.uk

X. Gu · S. Ramsay · M. Jensen · R. Fulton · S. Rosser University of Glasgow, University Avenue, Glasgow G12 8QQ, UK
}

and can be considered as a reference framework for the development of biochemical systems in synthetic biology.

Keywords Biosensor - Synthetic biology $\cdot$ Genetic engineering $\cdot$ Computational modelling

\section{Introduction}

The elimination of pollutants from the environment is essential for the sustainable development of our society. Interest in the microbial biodegradation of pollutants has intensified in recent years due to the high cost and environmentally destructive nature of conventional technologies. Bioremediation endeavours to harness the astonishing natural ability of microbial metabolism to degrade a vast range of important pollutants including hydrocarbons (e.g. oil) (Head et al. 2006), polychlorinated biphenyls (PCBs) (Pieper 2005), polyaromatic hydrocarbons (PAHs) (Samanta et al. 2002) and pharmaceutical substances (Halling-Sørensen et al. 1998). A greater public awareness of environmental issues has driven research into technologies that can detect changes in a wide range of environmental conditions, especially those which require continuous monitoring. An emerging strategy is to utilize living organisms engineered to produce a measurable output in response to specific pollutants in biosensors. The two most desirable features of a commercially viable, in situ biosensor are a biological sensing element which recognises the target pollutant and a reporter element which mediates both quantitative and qualitative measurements of pollutant levels in real time. The preponderance of aromatic degradation genes on mobile genetic elements has lead, via horizontal gene transfer, to the evolution of novel aromatic degradative pathways, common intermediates of which 
present us with a handle on the presence of diverse pollutants (Tan 1999). Conventional reporter elements, including green fluorescent protein (GFP), firefly/renilla luciferase and $\beta$-galactosidase (LacZ), are often expensive and involve technical expertise and laboratory-based assays (Rogers 2006).

In recognition of these problems, the aim of the Glasgow iGEM 2007 project was to develop a biosensor with a novel reporter mechanism to provide an inexpensive and easy-to-use method of detecting target analytes in real time, as the first step in the construction of a self-powered biosensor. The structure of this paper is as follows: we give an overview of the system requirements in Sect. 2, and describe the system-level design and associated computational methods Sect. 3. The construction of the system is presented in Sect. 4, and details of the "Materials and Methods" are given in Sect. 5. Finally we discuss some conclusions in Sect. 6.

\section{Requirements overview}

The discovery of transcriptional activators and their corresponding promoter sequences has made possible the development of bacterial biosensors for pollutants. Modified cell biosensors are constructed by fusing a reporter gene (an enzyme or a fluorescent protein e.g. GFP) to a promoter element that is induced by the presence of a target compound. In the presence of an organic contaminant the transcriptional activator changes its three dimensional structure, becoming operative, and transcription of the reporter gene is enhanced. The gene is transcribed to form mRNA which is then translated into a protein which performs the biochemical activity (in the case of enzymes) or fluoresces (e.g. GFP). The resulting increase in reporter gene product is then detected by measuring the activity of the reporter enzyme or the fluorescence of the reporter protein. Thus, under appropriate conditions, a direct correlation between contaminant concentration and reporter product can be established.

One holy grail of environmental biosensors is to create a system which can be left in the field continuously monitoring and remotely sending electronic signals back to a computer. One major problem is how to power such a device so that frequent expensive battery changes are not necessary. One possible source of renewable energy for powering biosensor devices are microbial fuel cells (MFC) in which micro-organisms oxidize compounds such as glucose, acetate or wastewater. The electrons gained from this oxidation are transferred to an electrode. In the past, external, expensive, soluble redox mediators have consistently been added to MFCs to enhance electron transfer. Pseudomonas aeruginosa has been shown to produce its own electron transporters, pyocyanin (PYO), which can function as electron-carrying redox mediators increasing electrical power output of MFCs.

Our project aimed to use a synthetic biology approach to combine the production of an environmental biosensor for economically important industrial environmental pollutants with a microbial fuel cell which can produce its own electricity. The intention is that the cells will recognise the presence of a pollutant via a modular interchangeable range of pollutant-specific transcriptional activator proteins and enhance electricity generation in a microbial fuel cell by inducing genes for the synthesis of the electron mediator PYO which function as novel reporter genes.

The recognition element of our 'ElectrEcoBlu' biosensor system is a pollutant responsive transcriptional activator, which can be either XylR or DntR, binding the following classes of important environmental pollutants: BTEX chemicals (benzene, toluene, ethylbenzene and xylene) and dinitrotoluene respectively. The reporter element of the biosensor consists of the enzymes $S$-adenosylmethioninedependent $N$-methyltransferase (PhzM) and flavindependent hydroxylase (PhzS) which convert the precursor compound phenazine-1-carboxamide (PCA) to PYO in the biosynthetic pathway cloned from Pseudomonas aeruginosa into E. coli and a non-pathogenic Pseudomonas strain.

\section{System design}

One of the central features of synthetic biology is the application of approaches from science and engineering disciplines to aid the design and understanding of synthetic biological machines. The Glasgow iGEM project explored the use of a variety of computational methodologies and developed tools to support the engineering of biosensors, prediction of systems dynamics, validation of biological hypothesis and the guiding of optimal experimental design.

The molecular biologists of our team constructed an initial diagram to describe the system, using a fairly informal graphical syntax, see Fig. 1a. The generic form of the transcription factor ('tf' for the gene, and ' $\mathrm{TF}$ ' for the protein product) represents both XylR (toluene detecting) and DntR (salicylate detecting). The ideas for system design (with and without the positive feedback loop) developed by the biological part of the team resulted in the initial design diagrams. Due to the complexity of the model and the lack of sufficient parameters required for numerical simulation in the later stage of the project, we decided to refine the initial system (Fig. 1a) by removing the mRNAs to assume a one-step reaction of gene transcription and translation, and merging the two genes $p h z S$ and $p h z M$ as a 


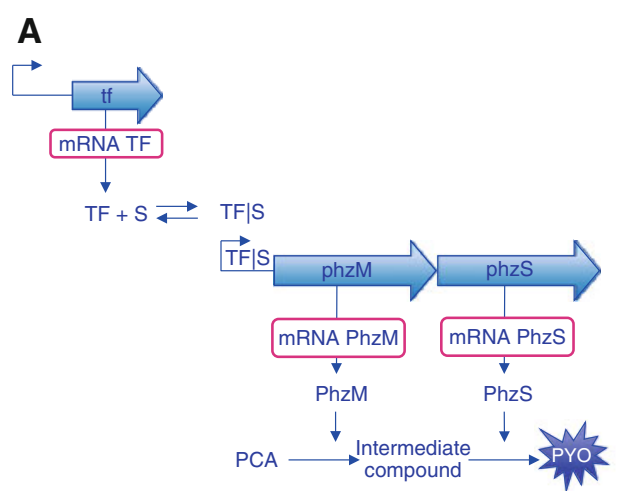

Fig. 1 'ElectrEcoBlu' design diagrams. a The original representation of the simple reporter system. b The refined topology of the reporter system with the positive feedback loop highlighted in yellow. TF stands for a generic transcriptional factor and $S$ stands for input

single entity $p h z M S$ which product catalyzes the conversion of PCA into pyocyanin, a simplification justified by the work of Parsons et al. (2007) who reported that they were not able to characterise PhzM and PhzS separately. Model refinement therefore allows us to reduce the complexity of the system without sacrificing essential components.

Using the refined model, we studied the behaviour of the basic reporter system and its variant featuring a positive feedback loop (Fig. 1b). We used an unified framework of qualitative, stochastic and continuous approaches (Fig. 2), based on Gilbert et al. (2007), as a basis for our modelling and analysis of the systems.

We firstly explored the use of Petri nets for the system construction and analysis. Petri nets provide an intuitive

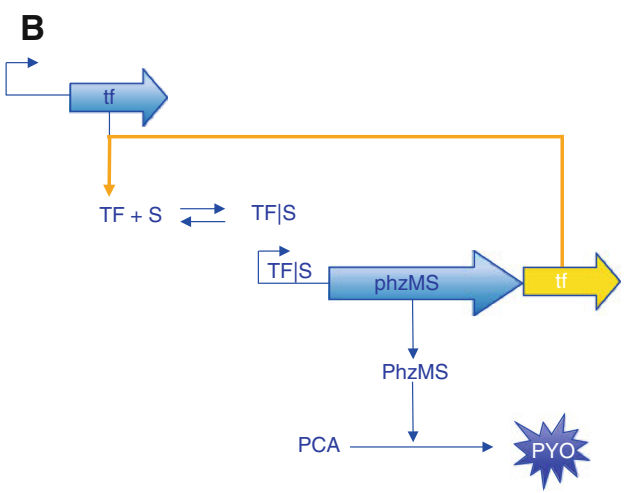

signal compound. The simple reporter system without the positive feedback loop can be obtained by removing the redundant TF and the loop (in yellow) attached after the thick arrow titled PhzMS

graphical representation of the system which can be understood by both biologists and modellers. We transformed the initial diagrams into a qualitative Petri-net model (Fig. 3) using the Snoopy tool (Rohr et al. 2010) and conducted an invariant analysis of the network properties using the Charlie program (Charlie 2010). The qualitative analysis is promising for describing and studying systems in the absence of reliable parameters required by quantitative approaches. Behavioural properties supported by Petri nets such as boundedness, liveness and reversibility can help us develop a sound model representation that can capture essential characteristics of the system. There are two types of nodes in Petri-net models, place nodes represented as circles, which stand for biochemical entities in the case of biochemical network models, and transition nodes standing

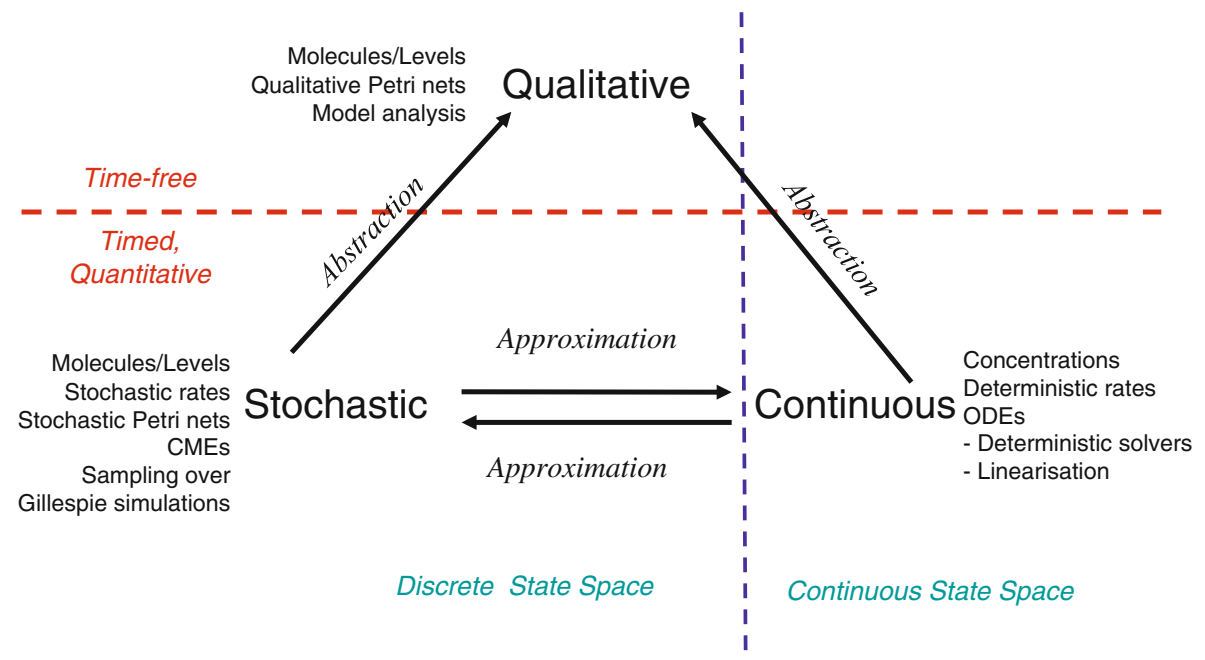

Fig. 2 Conceptual modelling framework. Qualitative representations are minimally described by the network topology, with nodes representing either reactions or species, and concentrations being described as discrete quantities. The stochastic approach associates a probabilistically distributed firing rate (waiting time) with each reaction. The continuous model replaces the discrete values of species with continuous values, and associates a particular deterministic rate information with each transition, permitting the continuous model to be represented as a set of ordinary differential equations (ODEs). Adapted from Gilbert et al. (2007) 


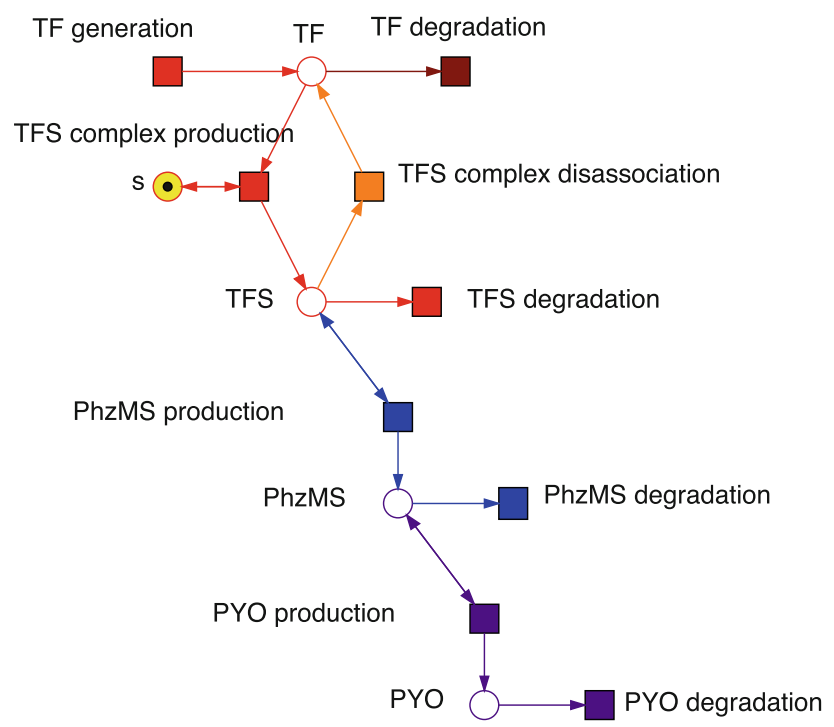

$1 \mathrm{P}$-invariant, 5 T-invariants

Fig. 3 Qualitative Petri Net model. Bipartite directed graph with nodes standing for biochemical entities (circles) or reactions (rectangles). $T F$ transcription factor, $S$ signal, TFS complex, PhzMS PhzM and PhzMS enzymes, $P Y O$ pyocyanin

for reactions, represented as boxes. Invariant analysis supported by Petri-net based modelling are Place invariants (or P-invariants), represented as sets of places where the sum of tokens remains constant over any firing, and Transition invariants (or T-invariants), represented as sets of transitions which have a zero effect on the marking of the system. If the T-Invariants cover the entire Petri net, it shows that the system can have a cyclic behaviour, while incorporating all system parts, which suggests that the system might have been modelled correctly. Briefly, the Petri net model for our design has the deadlock trap property (live, with no dead transitions or dead states), is not covered by P-invariants, but is covered by T-invariants ( 5 for the basic model with 2 additional for the feedback model). The occurrence of dynamic conflicts could not be decided by the analysis tool due to the unboundedness of the net, but by playing the token game we could easily observe the occurrence of such behaviour, where two transitions, sharing a pre-place, are both enabled, but the firing of one transition disables the other one.

In order to quantify the system's output, we conducted an intensive literature search to extract the rate parameters necessary for numerical simulation of the Ordinary Differential Equations (ODE) based modelling. The ODE equations that we have devised for our basic model are presented in Table 1 and Eqs. 1, 2, 3 and 4. In order to represent the positive feedback loop, the term $v_{10}$ has to be added to the Eq. 1 of the simple reporter model.
Table 1 ODE terms The processes of protein production and degradation, complex association and dissociation, and enzymatic reactions were modelled using the terms presented in this table

\begin{tabular}{lll}
\hline Index & Term & Explanation \\
\hline$v_{1}$ & $\alpha_{T F}$ & $T F$ production (constant) \\
$v_{2}$ & $\delta_{T F} \cdot T F$ & $T F$ degradation \\
$v_{3}$ & $k_{a} \cdot s \cdot T F$ & $T F$ and $s$ association into $T F S$ \\
$v_{4}$ & $k_{d} \cdot T F S$ & Dissociation of $T F S$ into $T F$ \\
$v_{5}$ & $\delta_{T F S} \cdot T F S$ & $T F S$ degradation \\
$v_{6}$ & $\beta_{P h z M S} \cdot \frac{T F S}{\gamma_{P h z S S}+T F S}$ & PhzMS production inducible by $T F S$ \\
$v_{7}$ & $\delta_{P h z M S} \cdot P h z M S$ & $P h z M S$ degradation \\
$v_{8}$ & $\alpha_{P Y O} \cdot P h z M S$ & Pyocyanin $(P Y O)$ production \\
$v_{9}$ & $\delta_{P Y O} \cdot P Y O$ & Pyocyanin $(P Y O)$ degradation \\
$v_{10}$ & $\beta_{T F} \frac{T F S}{\gamma_{T F}+T F S}$ & $T F$ production induced by $T F S$ \\
\hline
\end{tabular}

$\dot{T F}=v_{1}-v_{2}-v_{3}+v_{4}$

$\dot{T F S}=v_{3}-v_{4}-v_{5}$

PhzMS $=v_{6}-v_{7}$

$P \dot{Y} O=v_{8}-v_{9}$

The parameter values used in the model are presented in Table 2. Some of the values have a significant degree of uncertainty and are therefore assigned value ranges.

We have applied sensitivity analysis to identify the critical parameters of the system. A system is sensitive to a parameter if changes in the parameter's value significantly affect behaviour of the system. It is always the case that parameter values are affected by error. Because of inaccuracy of measurements, inaccuracy of the model or natural variability due to environmental conditions (temperature, etc.). Therefore it is often best to think of parameters as random variables and to test the system for its sensitivity to the variability.

Single parameter sensitivity analysis techniques give a very narrow insight as only one parameter is varied at a time while other remain fixed at some arbitrary values. Multi-parametric Sensitivity Analysis (MPSA) (Choi et al. 1999) is a Monte Carlo method in which the entire parameter space is sampled from distributions of the parameters. Usually, uniform distribution and Latin square sampling is used. The model is simulated for all of the parameter value samples and the output compared with a baseline output coming from experimental data or simply being the model output for parameter values at the centre point of the parameter space. Then, the samples are partitioned into two sets: 'acceptable' and 'unacceptable' according to the disagreement in output with the baseline. Cumulative distribution over each parameter range for both 'acceptable' and 'unacceptable' samples are plotted. The 
Table 2 Constant values used in the ordinary differential equation models

\begin{tabular}{|c|c|c|c|}
\hline Symbol & Value & Range & Comment \\
\hline$\alpha_{T F}$ & $0.07 \mu \mathrm{M} \cdot s^{-1}$ & $0.05-0.1$ & Average production rate \\
\hline$\beta_{T F}$ & $0.07 \mu \mathrm{M} \cdot s^{-1}$ & $0.05-0.1$ & Average production rate \\
\hline$\gamma_{T F}$ & $5 \mu \mathrm{M}$ & $0.1-10$ & From range of DNA-binding constants (e.g., Jung et al. 2005) \\
\hline$k_{a}$ & $1 \mathrm{e} 6(\mu \mathrm{M} \cdot s)^{-1}$ & & Greater than fastest known enzyme \\
\hline$k_{d}$ & $4 \mathrm{e} 6 s^{-1}$ & & A very fast reaction \\
\hline$\delta_{T F}$ & $3.851 \mathrm{e}-4 s^{-1}$ & $2.567 \mathrm{e}-4-5.776 \mathrm{e}-4$ & $\begin{array}{l}\text { Based on } 30 \text { min half life (range } 20-45 \text { for bacterial } \\
\text { transcription factors (e.g., Zhu and Winans 1999)) }\end{array}$ \\
\hline$\delta_{T F S}$ & $3.851 \mathrm{e}-4 s^{-1}$ & $2.567 \mathrm{e}-4-5.776 \mathrm{e}-4$ & $\begin{array}{l}\text { Range } 20-45 \text { for bacterial transcription factors } \\
\text { (e.g., Zhu and Winans 1999) }\end{array}$ \\
\hline$\beta_{P h z M S}$ & $0.1 \mu \mathrm{M} \cdot s^{-1}$ & & Standard rate for $300 \mathrm{aa}$ bacterial protein \\
\hline$\gamma_{P h z M S}$ & $5 \mu \mathrm{M}$ & $0.1-10$ & From range of DNA-binding constants e.g. (Jung et al. 2005). \\
\hline$\delta_{P h z M S}$ & $8.0225 \mathrm{e}-6 s^{-1}$ & & $\begin{array}{l}\text { Based on } 24 \text { h half life (Bacterial protein norm, } \\
\text { e.g., Nath and Koch 1970) }\end{array}$ \\
\hline$\alpha_{P Y O}$ & $1.3 s^{-1}$ & & Parsons et al. (2007) \\
\hline$\delta_{P Y O}$ & $5.8 \mathrm{e}-1 s^{-1}$ & & $\begin{array}{l}\text { In human cells (O'Malley et al. 2003). Probably } \\
\text { much faster in } E \text {. coli }\end{array}$ \\
\hline
\end{tabular}

Fig. 4 Multi-parametric Sensitivity Analysis. The graphs show cumulative distributions of 'acceptable' (solid line) and 'unacceptable' (dashed line) for several parameters in the ODE model at different value ranges. The $X$-axes represent rate parameter values, see Table 2 for descriptions and units; the signal $s$ is in $\mu \mathrm{M}$. The $Y$-axes refer to the number of acceptable and unacceptable samples tested, each normalised to the range $[0,1]$. The discrepancy between the lines indicates how sensitive the model is to each parameter. $\gamma_{P h z M S}$ is the most significant in the considered ranges
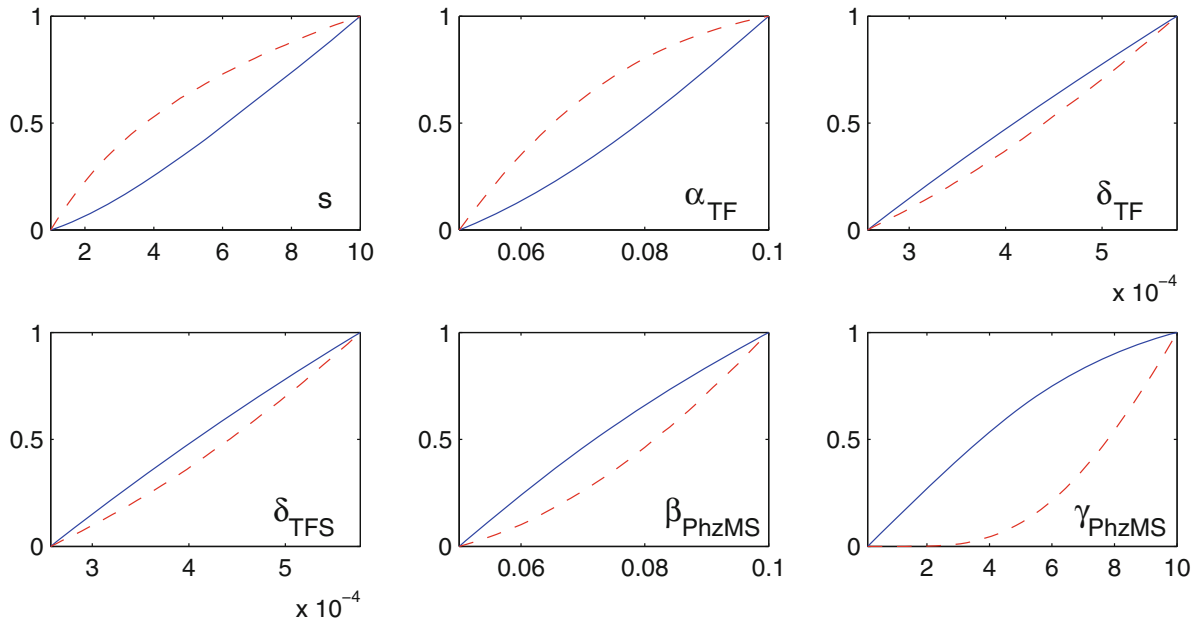

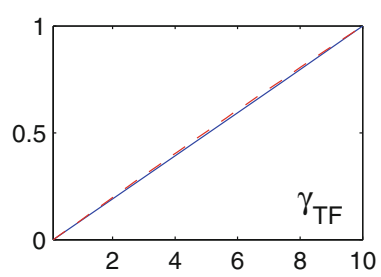

discrepancy between the two curves indicates that change in parameter's value affects the likelihood of samples being in large or small disagreement with the baseline and therefore that the parameter is significant.

Figure 4 shows output of a slightly modified MPSA that recognises parameters significant to the difference in behaviour between two models-the basic and feedback model in our case. No baseline system output is used-the disagreement between outputs of the two models is measured with the constraint that the parameters shared between the models have the same values for each comparison. In this run, we have explored parameters with the most uncertain values. Parameter $\gamma_{P h z M S}$ turned out to be the most sensitive one. Thus, in order to refine our comparison we would first try to narrow the value range for this parameter. Such information would let us prioritise costly and time consuming wet-lab measurements in further work.

Additionally, we developed a graphical modelling environment based on Simulink ${ }^{1}$ for component-based

\footnotetext{
${ }^{1}$ Simulink product web page: http://www.mathworks.com/products/ simulink
} 
ODE dynamic modelling of biological systems at the BioBrick level. The efforts involved constructing a library of mathematical functions which described the behaviour of the BioBricks. In doing so, we introduced a linear approximation of nonlinear elements using a transformation based on the frequency (Laplace) domain.

Modelling genetic systems in terms of ODEs is often not sufficient as cells are intrinsically noisy due to low numbers of molecules that participate in reactions. This can lead to significant statistical fluctuations in reaction rates and, the system's behaviour can differ from that predicted by a deterministic model. The Gillespie algorithm allows a discrete and stochastic simulation with few reactants because every reaction is individually simulated. It takes into account a number of parameters that contribute to the model in a random manner, rather than assuming everything can be predicted.

One goal of the project was to construct a stochastic model based on a deterministic one and to investigate the amount of noise, measured by the the coefficient of variation. A stochastic model for a general biosensor was constructed and simulated using our own implementation of the Gillespie algorithm (Gillespie 1977) in MATLAB. Simulations were performed for several of cells and repeated for many runs to find representative behaviour. Noise was expected to decrease as the number of cells increased (Fig. 5). We computed noise using the coefficient of variation, defined by
Table 3 Table of noise for increasing number of cells, as measured by the coefficient of variation

\begin{tabular}{lllll}
\hline No. of cells & 1 & 10 & 100 & 1000 \\
coeff var & 0.0642 & 0.0221 & 0.0136 & 0.0078 \\
\hline
\end{tabular}

coeff $\operatorname{var}=\frac{\sigma}{\mu}$,

where $\sigma$ is the standard deviation and, again, $\mu$ is the mean.

The results of our analysis, presented in Table 3 found that the coefficient of variation decreased as the number of cells increased. In practice our sensor will contain a very large number of cells (in the order of millions), and hence the biological noise will be negligible, and is not an important factor affecting the system implementation. We also aimed to study whether generic features predicted by other modelling approaches can be repeated by stochastic simulations. The effects of leakiness of the promoter was also observed in the system. Leakiness had a detrimental effect on the reliability of the system. It provides a higher output of the protein which in turn gives a false indication of the level of signal being detected.

\section{System construction}

We used the computational-based design described in the previous section to inform the construction of the
Fig. 5 Stochastic simulations. The figure (clockwise from the top left) displays the response of pyocyanin in number of molecules in response to a signal $s=10 \mu \mathrm{M}$ over time produced by simulations for 1 , 10,100 and 1,000 cells, averaged over 10 runs
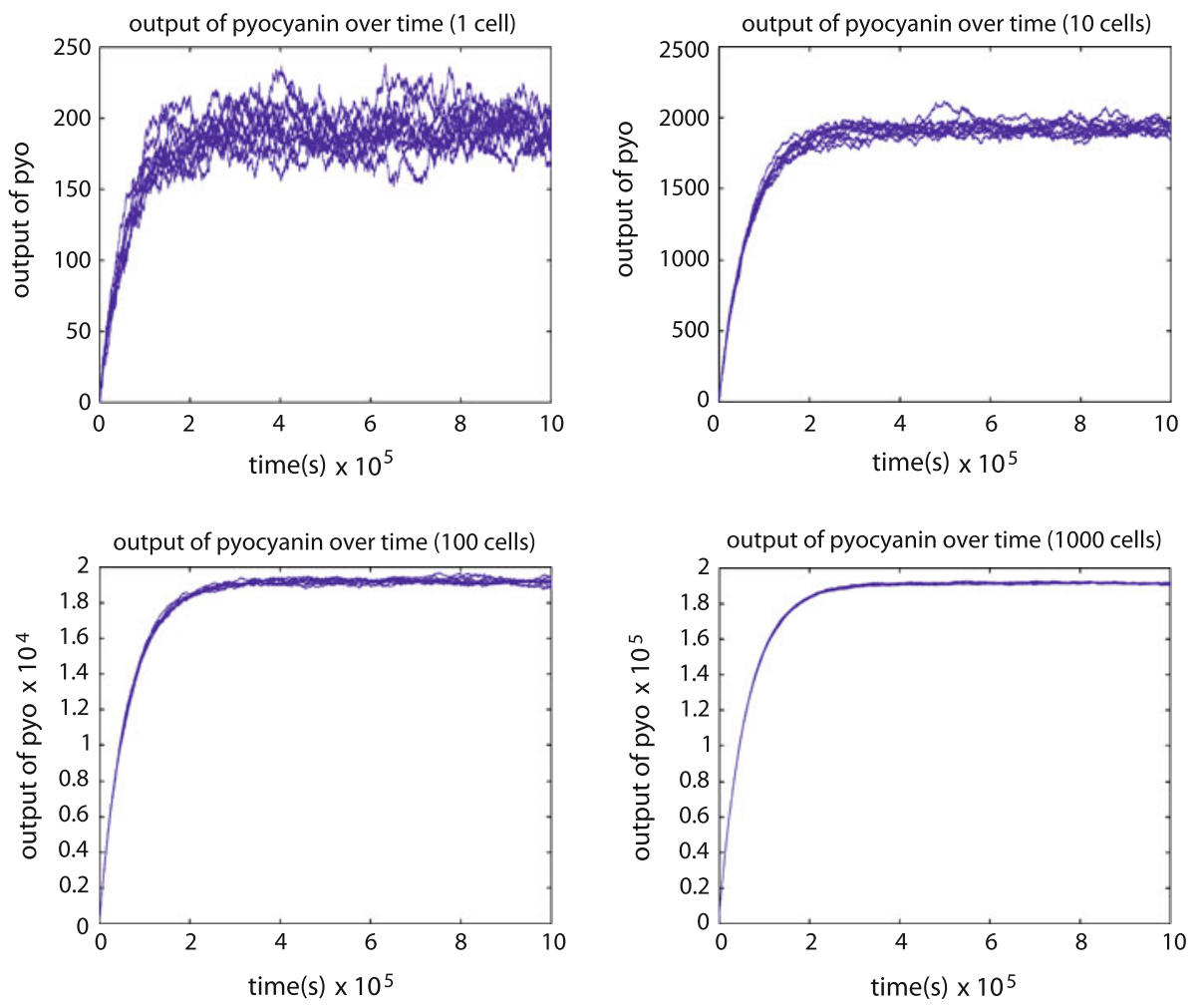
physical system. As shown in Fig. 1, ElectrEcoBlu consists of two components; the sensor element which is modular, permitting customisation to detect a range of input signals as required, and the reporter element which, on the other hand, is always responsible for generating an electrical signal as an output. Since the novel sensor and reporter modules were being developed in parallel, pre-existing LacZ and luciferase reporters were utilised to constitute variations of a complete biosensor in order to validate functionality of the sensing components constructed.

\section{The sensor}

During the course of the project, consideration was given to two different sensing constructs. The generic design of the sensor module consists of a constitutively expressed protein which undergoes a conformational change upon binding with a specific pollutant molecule. This complex then acts as a positive transcriptional regulator on a target promoter, activation of which leads to production of the reporter protein. In the rest of this section, we present the genetic constructs considered in response to the following classes of organic pollutants: BTEX chemicals (benzene, toluene, ethylbenzene and xylene) and dinitrotoluene (Rickert et al. 1984).

\section{XylR and BTEX chemicals}

Upon binding BTEX compounds the XylR protein (encoded on the TOL plasmid from Pseudomonas putida) recognises and activates the promoter $\mathrm{P}_{u}$ (Inouye et al. 1988). In order to provide a qualitative indication of the specificity of XylR for BTEX compounds, a Miller assay was conducted on a pQF52-derived construct provided by Dr. Susan Rosser, University of Glasgow, carrying the complete $x y l R$ coding and regulatory region directing the expression of LacZ under the control of $\mathrm{P}_{u}$. Highest levels of LacZ activity were observed in response to toluene and xylene (Fig. 6a). In order to produce a novel modular sensing component for BTEX compounds several genetic elements had to be sub-cloned into BioBrick format; the $x y l R$ coding region with its native promoter $\mathrm{P}_{r}$ and presumed RBS (Willardson et al. 1998), and the target promoter $\mathrm{P}_{u}$. These sequences were submitted to the Registry of Standard Biological Parts (see "Materials and Methods" for details and Registry numbers). These were assembled according to BioBrick specifications (Fig. 6b) along with a standard ribosome binding site (BBa_J61101) and a renilla luciferase coding region in (BBa_J52008) taken from the Registry to allow in-progress testing for sensitivity to toluene. By the end of the time allotted for the project a qualitative response was confirmed to $250 \mu \mathrm{M}$ toluene (Fig. 6c) in a luciferase assay. These results indicate that
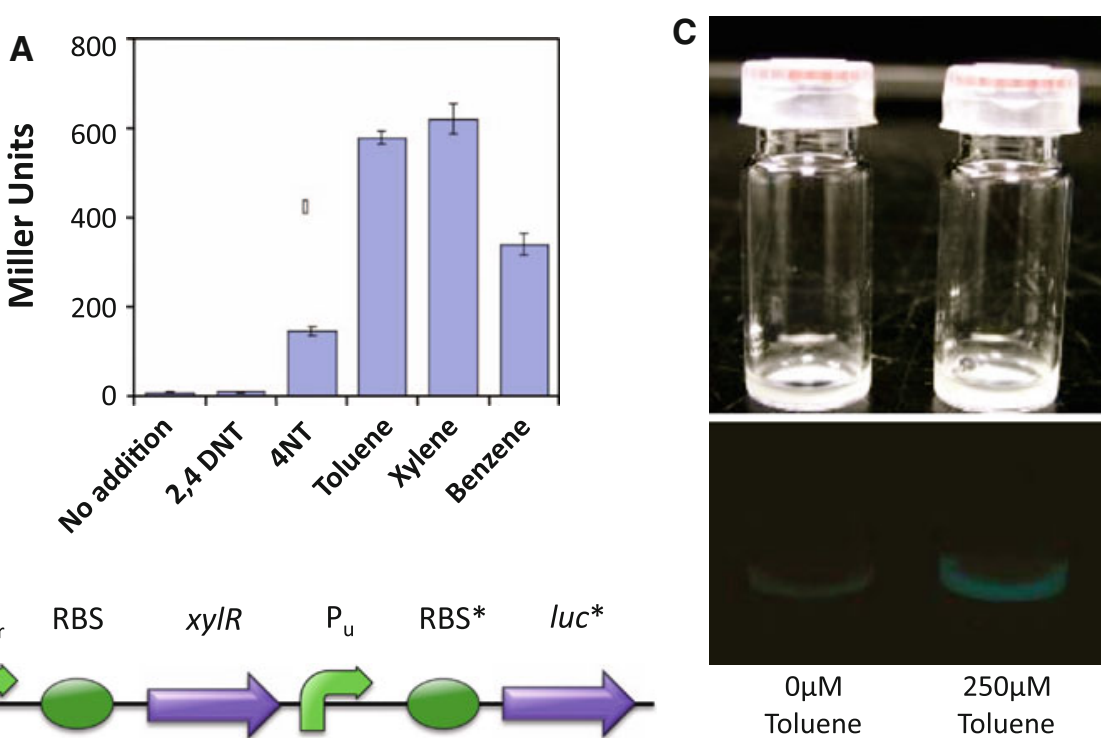

Fig. 6 The XylR sensor. a XylR-mediated sensitivity to, and selectivity for, BTEX compounds present in the extra-cellular medium as measured by expression of an existing LacZ reporter construct, quantified by the Miller Assay. b The construct created by the Glasgow iGEM team to test suitability of $x y l R$ as a modular sensor component, shown in standard BioBrick notation driving expression of luciferase. Genetic elements were cloned from the naturally- occurring TOL plasmid carried by Pseudomonas putida and (*) denotes BioBricks supplied by the Registry of Standard Biological Parts (see "Materials and Methods" for details). C $10 \mathrm{ml}$ vials containing $1 \mathrm{ml}$ of culture photographed in the light (upper panel) or in the dark (lower panel) with and without toluene. A qualitative difference in fluorescence was observed at a concentration of $250 \mu \mathrm{M}$ 
XylR can detect a range of BTEX compounds and that it contributes as predicted as a novel modular sensor component in a functional biosensor.

\section{DntR and dinitrotoluene}

The transcriptional regulator DntR, a member of the LysR transcription factor family isolated from a plasmid carried by the soil bacterium Burkholderia cepacia R34, drives low level expression from promoter $\mathrm{P}_{D N T}$ upon binding the industrial pollutant 2,4-dinitrotoluene (2,4-DNT) and high level expression on binding of the poly aromatic hydrocarbon degradation intermediate salycilate (Smirnova et al. 2004; Suen et al. 1996). In a prototype biosensor, DntR-driven GFP expression was induced at a low level in the presence of 2,4-DNT at a high level by also salicylic acid demonstrating that the natural selectivities had been retained (Smirnova et al. 2004), a breakdown intermediate of aromatic compounds in soil bacteria. This joint sensitivity provided us with an alternative pollutant intermediate with which to perform an assay that was still environmentally relevant while less hazardous to use in our undergraduate lab. In order to turn this system into a modular sensor for 2,4-DNT the complete $d n t R$ gene and its target promoter, which lie in tandem on the source plasmid, were sub-cloned from a pQF52-derived construct which carries the entire 2,4-DNT responsive operon into a single BioBrick to provide a complete functional sensor entity (Fig. 7a), and submitted to the Registry of Standard

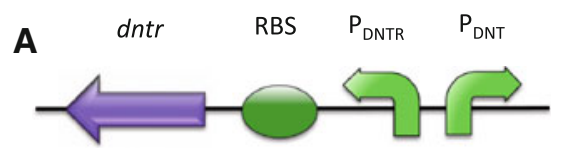

\section{B}

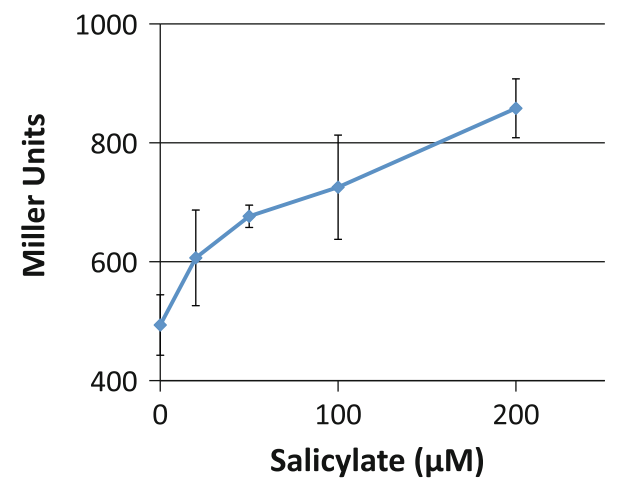

Fig. 7 The DntR Sensor. a The complete $d n t R$ gene and its target promoter, which lies in tandem and in the opposite orientation on the source DNA, were cloned as a single functional entity from a pQF52derived plasmid. b This source DNA included a lacZ reporter gene downstream of DntRs target $\mathrm{P}_{D N T}$ promoter; LacZ activity in E. coli carrying the construct was correlated with the concentration of salicylate added to the extracellular medium
Biological Parts. We conducted a Miller assay on the pQF52-derived plasmid to drive expression of lacZ to determine the potential of this detection system for indirect quantification of pollutants. A correlation was observed between LacZ activity and concentration of salicylate in the extra-cellular medium at concentrations between $20-200 \mu \mathrm{M}$ (Fig. 7b). Work is ongoing to assess the dose-response of our BioBrick to 2,4-DNT and to subclone the individual genetic components of the $d n t R$ region, sequences of which have also been individually deposited at the Registry. In order to improve DntR response to $2,4 \mathrm{DNT}$ it would be necessary to perform directed evolution on the dntR gene and select for 2,4DNT recognition.

The reporter

\section{Pyocyanin}

Our reporter mechanism is designed to harness the redox properties of the compound pyocyanin (Hassan and Fridovich 1979), an antibiotic secreted by Pseudomonas aeruginosa, to mediate electron transfer and thus to generate a current in a microbial fuel cell (MFC). When supplied exogenously, pyocyanin has been demonstrated to be utilised by other bacteria, resulting in an increase in their ability to transfer electrons to the anode of a MFC, thereby boosting the output signal (Rabaey et al. 2005); we therefore decided to clone the metabolic pathways necessary for its production to act as our inducible reporter component. The pyocyanin biosynthesis pathway from $P$. aeruginosa is encoded in two parts; a pair of functionally-redundant seven-gene operons ( $p h z A B C D E F G 1$ and 2) responsible for the synthesis of phenazine-1-carboxylic acid (PCA) (Mavrodi et al. 2001), and two enzymes encoded at distinct loci $(p h z M$ and $p h z S)$ which further modify PCA to produce pyocyanin (Parsons et al. 2007). In order to ensure a rapid response from the biosensor a constant pool of PCA should be maintained so that the time taken from sensor-activation to current generation relied only on the kinetics of transcription/translation of $p h z M$ and $p h z S$, and activity of their respective enzymes. To achieve this it was decided that phzABCDEFG 1 should be cloned under the control of a constitutive promoter and $p h z M$ and $p h z S$ each under the control of either of our modular inducible promoters; $\mathrm{P}_{u}$ (XylR-mediated BTEX response) or $\mathrm{P}_{D N T}$ (DntR-mediated 2,4-DNT response) (Fig. 8). Cloning of the $7 \mathrm{~kb} p h z A B C D E F G$ operon into a single BioBrick is on-going. Both $p h z M$ and $p h z S$ were cloned in BioBrick format and submitted to the Registry of Standard Biological Parts (BBa_I723024 and BBa_I723025, respectively). 


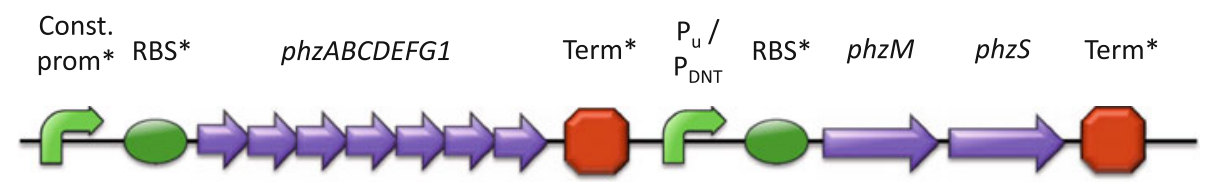

Fig. 8 The reporter construct design. One of the functionallyredundant phzABCDEFG operons which encode PCA-generating enzymes is shown here under the control of a constitutive promoter with a double-transcriptional-terminator signal; in tandem on the construct is the modular pollutant-responsive promoter $\left(\mathrm{P}_{u}\right.$ for XylRmediated responses; $\mathrm{P}_{D N T}$ for DntR-mediated responses) driving

\section{MFC measurements}

While cloning work was under way, small-scale MFCs (obtained from the National Centre for Biotechnology Education at the University of Reading) were set up using standard lab-strain E. coli (TOP10, Invitrogen) with no genetic modifications housed in the anode compartment (Fig. 9a). The NCBE MFC is a two-chamber Plexiglas system with the anode and cathode chambers physically separated by a polymeric proton exchange membrane (PEM) with an available surface area of $12 \mathrm{~cm}^{2}$ exposed to either compartment, and a working volume of $10 \mathrm{~cm}^{3}$ per compartment. The electrodes consisted of carbon fibre sheets. The cathode chamber contained a solution of potassium ferricyanide $0.02 \mathrm{M}$, in a potassium phosphate buffer $(\mathrm{pH}=7)$. The anode chamber contained the bacterial culture with a solution of methylene blue, and a small amount of glucose (0.5 M solution) (Morozan et al. 2007). In order to determine the range of electrical output we might expect from our finished system the production of pyocyanin had to be mimicked by adding a readily-available electron mediator. The dye Methylene Blue has a expression of phzM and phzS which encode the enzymes responsible for converting PCA to pyocyanin. (*) denotes BioBricks supplied by the Registry of Standard Biological Parts (see "Materials and Methods" for details). This diagram represents the DNA-level implementation of the design model without the feedback loop as shown in Fig. 1a

similar redox properties to pyocyanin so it was selected to be added to the $E$. coli culture in these preliminary experiments. A sustained electrical output of $500 \mathrm{mV}$ was observed for the $8 \mathrm{~h}$ following its addition, a clear improvement compared with readings of $150 \mathrm{mV}$ in MFCs lacking any electron mediator (Fig. 7b).

\section{Materials and methods}

Bacterial strains

\section{E.coli TOP10 Cells (Invitrogen)}

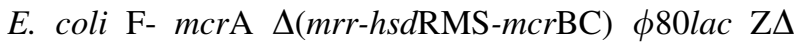
M15 $\Delta$ lac74 recA1 araD139 $\Delta$ (ara-leu) 7697 galU galK rpsL (StrR) endA1 nup $\mathrm{G} \lambda$ -

\section{Pseudomonas Strains}

Pseudomonas putida MT-2 carrying the TOL plasmid (Provided by Dr. Susan Rosser, University of Glasgow)

Pseudomonas aeruginosa PA01 (Provided by Dr. Dan Walker, University of Glasgow)
A

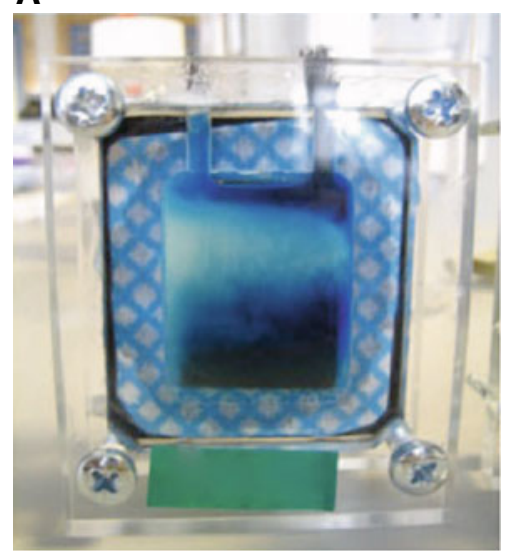

Fig. 9 Microbial fuel cell measurements. a A miniature microbial fuel cell (MFC) made of transparent plastic as viewed from the anode side. A suspension of untransformed E. coli is seen through the window after addition of methylene blue, a functional pyocyanin analog, with electrode wire protruding from a channel at the top. Fuel

B

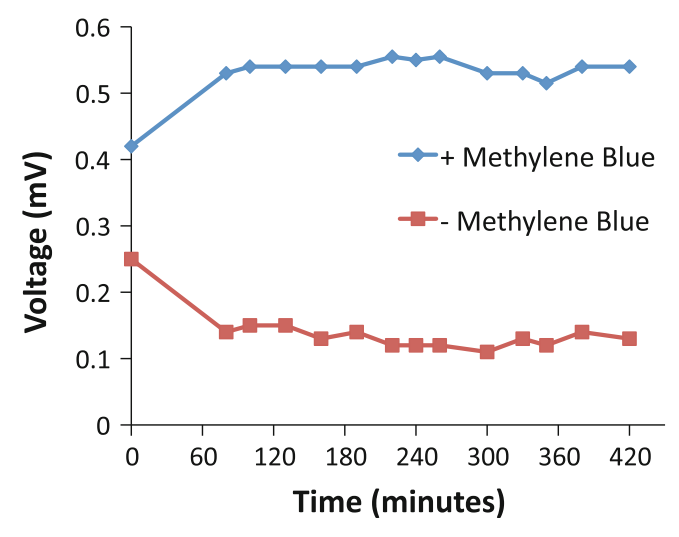

cell obtained from the National Centre for Biotechnology Education at the University of Reading). b Voltage readings taken from this MFC over the course of a day demonstrating the capability of our biosensor design to generate a measurable electrical output 
Table 4 Primer sequences

\begin{tabular}{|c|c|c|}
\hline Target & Type & Sequence \\
\hline \multirow[t]{2}{*}{$d n t R \mathrm{CDS}$} & Fwd & gaattcgcggccgcttctagATGGATCTGCGCGACATCG \\
\hline & Rev & ctgcagcggcegctactagtaTTATGCTTCAGAGAAAAGCTCG \\
\hline \multirow[t]{2}{*}{$d n t R+\mathrm{P}_{D N T}$} & Fwd & gaattcgcggecgcttctagGCAAGCTCTTTTTTCAGTTGTCTC \\
\hline & Rev & ctgcagcggcegctactagtaTTATGCTTCAGAGAAAAGCTCG \\
\hline \multirow[t]{2}{*}{$x y l R+\mathrm{P}_{r}$} & Fwd & gaattcgcggecgettctagAGATTTTAATGTGGGCTGCTTGGTG \\
\hline & Rev & ctgcagcggccgctactagtaCTATCGGCCCATTGCTTTCAC \\
\hline \multirow[t]{2}{*}{$\mathrm{P}_{u}$} & Fwd & gaattcgcggecgcttctagAGCCCGGGAAAGCGCGATGAAC \\
\hline & Rev & ctgcagcggecgctactagtaGGCGTAACGGCTACGGCTGGC \\
\hline \multirow[t]{2}{*}{$\operatorname{phz} M$} & Fwd & gaattcgcggecgettctagATGAATAATTCGAATCTTGCTGCT \\
\hline & Rev & ctgcagcggccgctactagtaTCAGGCCCTGGCAGCGAC \\
\hline \multirow[t]{2}{*}{$p h z S$} & Fwd & gaattcgcggccgcttctagATGAGCGAACCCATCGATAT \\
\hline & $\operatorname{Rev}$ & ctgcagcggcegctactagtaTTTTTTGCATGCTAGCGTGGCCGTTCC \\
\hline
\end{tabular}

Primers

All sequences were amplified using KOD hotstart polymerase (Novagen, catalog \#71086) according to the manufacturer's instructions with a final elongation step at $70^{\circ} \mathrm{C}$ for $2 \mathrm{~min}$. Initially all PCR products were cloned into $\mathrm{pCR}^{\circledR}$ 2.1-TOPO ${ }^{\circledR}$ vector (Invitrogen) and sequenced. The primers used are shown in Table 4. Once cloned and confirmed by sequencing all genes were digested with the appropriate restriction enzymes (Roche), following instructions in the Registry of Standard Biological Parts, prior to ligation into the BioBrick construction vectors using T4 DNA ligase (NEB).

\section{BioBrick assembly}

PCR products were ligated into construction vectors from the iGEM kit according to instructions online at the Registry of Standard Biological Parts.

\section{BioBricks used}

pSB1A2 construction vector (BioBrick ref BBa_p1010), pSB1AK3 construction vector (BioBrick ref BBa_I52001), Luciferase 2-monooxygenase from Renilla reformis (BioBrick ref BBa_J52008), Standard ribosome binding site (BioBrick ref BBa_J61101), Double rho-independent terminator (BioBrick ref BBa_B0015).

\section{BioBricks submitted}

Promoter $\mathrm{P}_{u}$ activated by XylR (BioBrick ref $\mathrm{BBa}$ I723020), Luciferase reporter activated by toluene (BioBrick ref BBa_I723023), PhzM coding region (BioBrick ref BBa_I723024), PhzS coding region (BioBrick ref $\mathrm{BBa}$ I723025), PhzM coding region with double terminators (BioBrick ref BBa_I723026), PhzS coding region with double terminators (BioBrick ref BBa_I723027), $\mathrm{P}_{u}$ with standard ribosome binding site (BioBrick ref BBa_I72 3029), DntR with double terminator BioBrick ref $\mathrm{BBa} \_$I723030), $\mathrm{P}_{u}$ with standard ribosome binding site followed by luciferase (BioBrick ref BBa_I723031), XylR coding region with native promoter $\mathrm{P}_{r}$ (BioBrick ref BBa_I723032).

Assays

Miller assay (modified from Zhang and Bremer (1995))

E. coli carrying either pQF52:dntR or pQF52:xylR were grown overnight in LB broth $(1 \%(\mathrm{w} / \mathrm{v})$ tryptone, $1 \%(\mathrm{w} / \mathrm{v})$ $\mathrm{NaCl}, 0.5 \%(\mathrm{w} / \mathrm{v})$ yeast extract, $\mathrm{pH} 7.0)$ or minimal media (0.6\%(w/v) Na2HPO4, 0.3\%(w/v) KH2PO4, 0.05\% (w/v) $\mathrm{NaCl}, 1 \mathrm{mM} \mathrm{MgSO} 4,200 \mathrm{mM} \mathrm{CaCl}$, $40 \mathrm{mg} / \mathrm{ml}$ thiamine, $1 \%(\mathrm{v} / \mathrm{v})$ trace elements, $3 \%$ succinic acid, $1 \mathrm{~g} / \mathrm{l} \mathrm{NH} 4 \mathrm{Cl})$ at $37^{\circ} \mathrm{C}$ with agitation to an $\mathrm{OD}_{600}$ of $0.28-0.7$.

Salicylate (final concentration: 0, 20, 50, 100, and $200 \mu \mathrm{M}$ ) or a variety of environmental pollutants (each to $0.25 \mathrm{mM}$ ) were added to the cultures which and were incubated at $37^{\circ} \mathrm{C}$ with agitation for a further $3 \mathrm{~h}$ to allow recognition by sensor proteins and expression of lacZ. The $\mathrm{OD}_{600}$ of each sample was noted and $20 \mu \mathrm{l}$ of culture was added to $80 \mu \mathrm{l}$ of permeabilisation solution $(20 \mathrm{mM} \mathrm{KCl}$, 100 mM Na2HPO4, 2 mM MgSO4, 0.8 mg/ml CTAB, 0.4 $\mathrm{mg} / \mathrm{ml}$ sodium deoxycholate, $5.4 \mu \mathrm{l} / \mathrm{ml} \beta$-mercaptoethanol) in $1.5 \mathrm{ml}$ microfuge tubes.

The samples and the substrate solution $(60 \mathrm{mM} \mathrm{Na} 2 \mathrm{H}$ PO4, $40 \mathrm{mM}$ NaH2PO4, $1 \mathrm{mg} / \mathrm{ml}$ o-nitrophenyl- $\beta$-DGalactoside (ONPG) $2.7 \mu \mathrm{l} / \mathrm{ml} \beta$-mercaptoethanol) were heated to $30^{\circ} \mathrm{C}$ in a water bath for $20-30 \mathrm{~min}$. Samples were then mixed with $600 \mu \mathrm{l}$ of substrate solution and incubated for different lengths of time before addition of $700 \mu \mathrm{l}$ stop solution (1 M Na2CO3) with thorough mixing. Samples were centrifuged at top speed in a bench top 
microfuge for 5-10 min. One milli litter of supernatant was transferred to a plastic cuvette and asborbance was measured at $420 \mathrm{~nm}$; Miller units were calculated using Formula 6.

$$
\frac{1000 \cdot A_{420}}{O D_{600} \cdot(\text { volume } 0.02 \mathrm{ml}) \cdot(\text { reaction time in } \operatorname{mins})}
$$

\section{Renilla luciferase assay}

This was performed using solutions supplied with the Renilla Luciferase Assay System (Promega, catalog \# E2810). E. coli carrying pSB1A2: xylR:luc were grown overnight at $37^{\circ} \mathrm{C}$ with agitation. The following day aliquots were taken and toluene added (final concentration: 0 or $250 \mathrm{mM}$ ) followed by further incubation at $37^{\circ} \mathrm{C}$ for a further $3.5 \mathrm{~h}$ to allow recognition by sensor proteins and expression of luciferase. Cell suspensions were centrifuged for $10 \mathrm{~min}$ at $3,000 \mathrm{rpm}$ followed by removal of supernatant. The cell pellet was then washed with $250 \mu 11 \times$ PBS and centrifuged again at $8,000 \mathrm{rpm}$ for $5 \mathrm{~min}$ after which the supernatant was removed. The pellet was suspended in $250 \mu 11 \times$ cell lysis buffer and freeze/thawed twice using liquid nitrogen. Luciferase assay reagent was prepared according to the manufacturer's instructions and $100 \mu \mathrm{l}$ per reaction was aliquotted into sealable glass vials followed by $20 \mu \mathrm{l}$ of lysate for each condition. Photographs were taken during the following $10 \mathrm{~min}$ in light and dark conditions to determine any visual differences in luminescence, as time constraints imposed by the iGEM project rules meant we were unable to quantify the output with a luminometer.

\section{Conclusions}

We have described the design and construction of a novel biosensor, using a design approach based on computational models, and investigated how to combine the biosensor with a microbial fuel cell. We also contributed a library of new high-quality BioBricks to the registry, allowing other researchers to adapt our work.

During the project we put into practice the application of a variety of computational modelling approaches with the aim of providing scientific explanations of the behaviours of the target systems and guidelines for optimal experimental design. We developed two designs, without and with feedback control, but due to time constraints only the basic model without feedback was actually developed.

In order to enable the systems to be further investigated and extended, we have created SBML models for our designs that can be imported and analysed by software tools compatible with the SBML format. All the materials that we have developed-models, software and tools—as well as descriptions of our protocols, sequences and BioBricks, and accompanying technical reports are available at the Glasgow 2007 iGEM wiki page (http://parts.mit.edu/igem07/index. php/Glasgow).

\section{Glasgow iGEM 2007 team}

The Glasgow iGEM 2007 Team consisted of 11 undergraduates from various academic backgrounds: Mai-Britt Jensen, Lynsey McLeay, Christine Merrick, Maija Paakkunainen and Scott Ramsay-Molecular Biology, Toby Friend and Christine Harkness-Mechanical Engineering, Karolis Kidykas-Aerospace Engineering, Rachael Fulton and Martina Marbá-Statistics, and Maciej Trybiło-Computing Science. The majority of the work was carried out during 10 weeks over the summer in an integrated laboratory environment. ElectrEcoBlu earned Glasgow a gold medal for their contributions to the nascent field of synthetic biology, and first prize in the Environment and Sensing category at iGEM 2007.

Acknowledgements The Glasgow iGEM team gratefully acknowledges financial support from the Glasgow University, Scottish Enterprise, the Eidgenössische Technische Hochschule (ETH) Zürich and the ANACHEM. We also would like to thank all the supporting academics-Dr. Emma Travis, Prof Raya Khanin, Dr. David Leader, Dr. David Forehand, Dr. Gary Gray, and Dr. Gabriela Kalna for their thought-provoking comments on the project. We are deeply indebted to Professor Monika Heiner for the constructive discussions on the Petri Nets based modelling approach.

The experiments performed in this study comply with the current laws of the United Kingdom. The authors declare that they have no conflict of interest.

Open Access This article is distributed under the terms of the Creative Commons Attribution Noncommercial License which permits any noncommercial use, distribution, and reproduction in any medium, provided the original author(s) and source are credited.

\section{References}

Charlie D (2010) A tool to analyse place/transition Petri nets. BTU Cottbus, CS Department. www-dssz.informatik.tu-cottbus.de

Choi J, Harvey JW, Conklin MH (1999) Use of multi-parameter sensitivity analysis to determ-ine relative importance of factors influencing natural attenuation of mining contaminants. In: Proceedings of the technical meeting, charleston, south Carolina, March 8-12, vol 1

Gilbert D, Heiner M, Lehrack S (2007) A unifying framework for modelling and analysing biochemical pathways using petri nets. In: In proceedings computational methods in systems biology CMSB 2007, vol. 4696. Springer, LNCS/LNBI, pp 200-216

Gillespie DT (1977) Exact stochastic simulation of coupled chemical reactions. J Phys Chem 81(25):2340-2361

Halling-Sørensen B, Nielsen SN, Lanzky PF, Ingerslev F, Lützhøft $\mathrm{HCH}$, Jørgensen SE (1998) Occurrence, fate and effects of pharmaceutical substances in the environment-a review. Chemosphere 36(2):357-393 
Hassan HM, Fridovich I (1979) Intracellular production of superoxide radical and of hydrogen peroxide by redox active compounds. Arch Biochem Biophys 196(2):385-395

Head IM, Jones DM, Röling WFM (2006) Marine microorganisms make a meal of oil. Nat Rev Microbiol 4(3):173-182. doi: 10.1038/nrmicro1348

Inouye S, Nakazawa A, Nakazawa T (1988) Nucleotide sequence of the regulatory gene $x y l R$ of the TOL plasmid from Pseudomonas putida. Gene 66(2):301-306

Jung KC, Rhee HS, Park CH, Yang CH (2005) Determination of the dissociation constants for recombinant c-myc, max, and dna complexes: the inhibitory effect of linoleic acid on the dnabinding step. Biochem Biophys Res Commun 334(1):269-275

Mavrodi DV, Bonsall RF, Delaney SM, Soule MJ, Phillips G, Thomashow LS (2001) Functional analysis of genes for biosynthesis of pyocyanin and phenazine-1-carboxamide from Pseudomonas aeruginosa PAO1. J Bacteriol 183(21):6454-6465. doi: 10.1128/JB.183.21.6454-6465.2001

Morozan A, Stamatin I, Stamatin L (2007) Carbon electrodes for microbial fuel cells. J Optoelectron Adv Mat 9:221-224

Nath K, Koch AL (1970) Protein degradation in Escherichia coli. I. Measurement of rapidly and slowly decaying components. J Biol Chem 245(11):2889-2900

O’Malley YQ, Abdalla MY, McCormick ML, Reszka KJ, Denning GM, Britigan BE (2003) Subcellular localization of Pseudomonas pyocyanin cytotoxicity in human lung epithelial cells. Am J Physiol Lung Cell Mol Physiol 284(2):L420-430. doi:10.1152/ ajplung.00316.2002. URL http://ajplung.physiology.org/cgi/con tent/abstract/284/2/L420

Parsons JF, Greenhagen BT, Shi K, Calabrese K, Robinson H, Ladner JE (2007) Structural and functional analysis of the pyocyanin biosynthetic protein PhzM from Pseudomonas aeruginosa. Biochemistry 46(7):1821-1828

Pieper DH (2005) Aerobic degradation of polychlorinated biphenyls. Appl Microbiol Biotechnol 67(2):170-191 doi:10.1007/s00253004-1810-4

Rabaey K, Boon N, Höfte M, Verstraete W (2005) Microbial phenazine production enhances electron transfer in biofuel cells. Environ Sci Technol 39(9):3401-3408
Registry of the Biological Parts, MIT (2010) Standard Assembly. URL http://partsregistry.org/Assembly:Standard_assembly (accessed $1 / 07 / 2010)$

Rickert DE, Butterworth BE, Popp JA (1984) Dinitrotoluene: acute toxicity, oncogenicity, genotoxicity, and metabolism. Crit Rev Toxicol 13(3):217-234 doi:10.3109/10408448409003373

Rogers KR (2006) Recent advances in biosensor techniques for environmental monitoring. Anal Chim Acta 568(1-2):222-231 doi:10.1016/j.aca.2005.12.067

Rohr C, Marwan W, Heiner M (2010) Snoopy-a unifying Petri net framework to investigate biomolecular networks. Bioinformatics 26(7):974-975

Samanta SK, Singh OV, Jain RK (2002) Polycyclic aromatic hydrocarbons: environmental pollution and bioremediation. Trends Biotechnol 20(6):243-248

Smirnova IA, Dian C, Leonard GA, McSweeney S, Birse D, Brzezinski P (2004) Development of a bacterial biosensor for nitrotoluenes: the crystal structure of the transcriptional regulator DntR. J Mol Biol 340(3):405-418 doi:10.1016/j.jmb.2004. 04.071

Suen WC, Haigler BE, Spain JC (1996) 2,4-Dinitrotoluene dioxygenase from Burkholderia sp. strain DNT: similarity to naphthalene dioxygenase. J Bacteriol 178(16):4926-4934

Tan HM (1999) Bacterial catabolic transposons. Appl Microbiol Biotechnol 51(1):1-12

Willardson BM, Wilkins JF, Rand TA, Schupp JM, Hill KK, Keim P, Jackson PJ (1998) Development and testing of a bacterial biosensor for toluene-based environmental contaminants. Appl Environ Microbiol 64(3):1006-1012

Zhang X, Bremer H (1995) Control of the Escherichia coli rrnB P1 promoter strength by ppGpp. J Biol Chem 270(19):11,181-11,189

Zhu J, Winans SC (1999) Autoinducer binding by the quorum-sensing regulator trar increases affinity for target promoters in vitro and decreases trar turnover rates in whole cells. Proc Natl Acad Sci USA 96(9):4832-4837 